\title{
Comparação entre diferentes métodos de inseminação artificial em suínos
}

\author{
Comparison between different methods of artificial insemination in swine
}

\author{
Luciano Auri dos Santos Flores ${ }^{1}$ Ivo Wentz ${ }^{2}$ Fernando Pandolfo Bortolozzo ${ }^{2}$ \\ Guilherme Borchardt Neto ${ }^{3}$ Rogério Francisco Balestrim ${ }^{4}$ Giuliano Gava ${ }^{4}$ \\ Rafael Kummer ${ }^{5}$
}

\section{RESUMO}

O objetivo deste trabalho foi avaliar três métodos de inseminação artificial(IA) no suíno em relação ao tempo de infusão e perdas por refluxo da DI durante a IA e aos 120 minutos após a IA, bem como dados de operacionalidade medido pelo grau de dificuldade verificado para execução da IA e dados de desempenho reprodutivo. Foram utilizadas 604 matrizes até a parição 7 e IDE $\leq 7$ dias. As fêmeas foram inseminadas em 3 tratamentos: $T 1=$ método auto IA com pipetas longas lameladas (Supertip ${ }^{\circledR}$ ), sêmen acondicionado em flexitubos ${ }^{\circledR}$ e aparato de IA constituído por uma cinta abdominal e uma mala dorsal; $T 2=$ método intermediário, pipetas Supertip ${ }^{\circledR}$, flexitubos ${ }^{\circledR}$ e sem aparato de IA; T3= método tradicional, pipetas tipo Melrose, sêmen acondicionado em bisnagas e sem aparato de IA, onde o tempo de IA ficou atrelado ao desempenho do funcionário. $O$ tempo médio de IA foi diferente $(P<0,02)$ entre os três tratamentos $(1,7 \pm 1,6 ; 2,2 \pm 1,8$ e 3,6 $\pm 1,1$ minutos para T1, T2 e T3 respectivamente). Em uma parte dos animais (108 fêmeas distribuídas nos 3 tratamentos) foram coletados os refluxos até 120 minutos transcorridos das IAs por meio de uma bolsa de colostomia fixada na vulva. O volume de refluxo durante a IA foi maior $(P<0,02)$ em $T 1 \quad(7,7 \pm 13,5 m L)$ quando comparado a T3 $(5,8 \pm 10,8 \mathrm{~mL})$. Não foram verificadas diferenças entre os tratamentos no número de espermatozóides eliminados por refluxo até 120 minutos após a IA. Independentemente do tratamento, em torno de $70 \%$ do volume e $30 \%$ do total de espermatozóides contidos na DI foram eliminados por refluxo em até duas horas após a IA. Na avaliação do grau de dificuldade, foi verificado que, no $T 1=85,6 \%$, no $T 2=92,9$ e no $T 3=97,7 \%$ das fêmeas tiveram suas inseminações concluídas com até uma intervenção ( $P<0,05$ entre todos os tratamentos). Com relação as taxas de retornos ao estro, taxa de parto ajustada e número de leitões nascidos não foram observadas diferenças entre os tratamentos $(P>0,05)$. As taxas de retorno ao estro foram 10,3, 7,4 e 8,5\%, a taxa de parto ajustada foi de 90,8, 94,0 e 91,7\% com 10,9, 11,1 e 11,1 leitões nascidos totais em T1, T2 e T3, respectivamente. $O$ método auto IA e o método intermediário proporcionam IAs mais rápidas e podem substituir o método tradicional sem prejuízos ao desempenho reprodutivo.

Palavras-chave: suínos, inseminação artificial, auto IA, refluxo, tempo de inseminação.

\section{ABSTRACT}

This study was performed to compare the Handsfree insemination method, i.e., the high degree automation procedure, with an intermediate method, which allows less participation of the inseminator during the application of the inseminating dose (ID), and the conventional method, which is widely used in Brazil. The comparison was based on infusion time and losses due to semen backflow during AI and 120 minutes after AI, degree of difficulty experienced during AI procedure, and reproductive performance data. A number of 604 sows on the parity up to 7 and a weaning to estrus interval $\leq 7$ days were used. Females were distributed into 3 treatments: $T 1=$ handsfree AI method, with long coilable shaft pipettes (Supertip ${ }^{\circledR}$ ), semen stored in flexitubes ${ }^{\circledR}$ and AI device consisting of an abdominal belt and a saddlebag: $T 2=$ intermediate method Supertip ${ }^{\circledR}$ pipettes, flexitubes ${ }^{\circledR}$, without AI device; T3= traditional method, Melrose-type pipettes, semen stored in tubs, without AI device. AI time was associated with the inseminator's performance. The average AI time was different $(P<0.02)$ among treatments $(1.7 \pm 1.6 ; 2.2 \pm 1.8$ e $3.6 \pm 1.1$ minutes for $T 1, T 2$ and $T 3$ respectively). One hundred eight sows distributed into the three treatments, had the semen backflow collected up to 120 minutes after AI in a colostomy bag fixed by the vulva. Semen backflow volume during AI was higher $(P<0.02)$ in $T 1(7.7 \pm 13.5$ $m L)$ as compared to $T 3(5.8 \pm 10.8 \mathrm{~mL})$. There were no differences among treatments in the number of spermatozoa eliminated by semen backflow up to 120 minutes after AI. Regardless of the treatment, nearly $70 \%$ of the volume and $30 \%$ of the total spermatozoa contained in the ID were eliminated by semen

${ }^{1}$ Médico Veterinário, Mestre, Faculdade de Veterinária, Universidade Federal do Rio Grande do Sul (UFRGS), Setor de Suínos, Porto Alegre, RS. Bolsista da Coordenação de Aperfeiçoamento de Pessoal de Nível Superior (CAPES).

${ }^{2}$ Médico Veterinário, Doutor, Faculdade de Veterinária, UFRGS, Setor de Suínos Setor de Suínos, Av. Bento Gonçalves 9090, 91540-000, Porto Alegre, RS. Autor para correspondência. E-mail: ivowentz@ ufrgs.br. Bolsista do Conselho Nacional de Desenvolvimento Científico e Tecnológico (CNPq).

${ }^{3}$ Médico Veterinário, Doutor, Universidade de Cruz Alta, Cruz Alta, RS.

${ }^{4}$ Acadêmico de Veterinária, Universidade Federal de Santa Maria, Santa Maria, RS.

${ }^{5}$ Acadêmico de Veterinária, UFRGS. Bolsista de Iniciação Científica, CNPq. 
backflow up to 2 hours after AI. Concerning the degree of difficulty of the AI procedure, $85.6 \%, 92.9$ and $97.7 \%$ of T1, T2 and T3 females were inseminated with up to 1 intervention $(P<0,05$ between all treatments). There were no differences among treatments $(P>0.05)$ in return to estrus rate, adjusted farrowing rate and number of born piglets. The return to estrus rates were 10.3, 7.4 and $8.5 \%$, adjusted farrowing rates were 90.8, 94.0 and $91.7 \%$, with 10.9, 11.1 e 11.1 total born piglets in T1, T2 and T3, respectively. The Hands free method and the intermediate method allowed faster AI and can replace the traditional method without influence on the reproductive performance.

Key words: swine, artificial insemination, Hands-free AI, semen backflow, insemination time.

\section{INTRODUÇÃO}

A inseminação artificial (IA) pelo método tradicional é amplamente difundida, demandando tempo e grande envolvimento de funcionários. Inovações e variações nos procedimentos de IA têm sido utilizadas para reduzir a mão-de-obra e o conseqüente custo de produção (BORTOLOZZO \& WENTZ, 1995).

Um aumento da automação na IA tem sido propost 0 com o desenvolvimento de diferentes tipos de pipetas, cintos e suportes para IA e diferentes embalagens para armazenar as doses inseminantes, mudanças essas que promovem variações, tanto na intensidade de estimulação da fêmea, como diferenças substanciais no próprio procedimento tradicional de IA. MARTIN RILLO et al. (1998) avaliaram o comportamento individual durante a aplicação de um método de auto-inseminação (auto IA), e observaram que as leitoas manifestaram uma excelente tolerância ao método, com uma variação no tempo de inseminação de 1,7 a 10,5 minutos. No mesmo trabalho, em um segundo experimento, MARTIN RILLO et al. (1998) avaliaram a fertilidade de 320 porcas inseminadas no método tradicional e por um sistema de auto IA, e encontraram uma taxa de parto de 83,75 vs $80,6 \%$ e 10,8 vs 10,5 leitões nascidos vivos para o método auto IA e método tradicional, respectivamente. Entretanto, até o momento, poucos estudos foram realizados para comparar a eficácia dessas novas tecnologias com o procedimento tradicional de IA utilizado em granjas tecnificadas.

O objetivo do trabalho foi comparar a aplicabilidade do método da auto IA, uma variação do mesmo, ou intermediário, e o método tradicional nas condições de rotina da granja, visando à otimização do tempo de IA/fêmea, as dificuldades de aplicação e o desempenho reprodutivo das fêmeas inseminadas.

\section{MATERIAL E MÉTODOS}

O trabalho foi realizado em uma granja multiplicadora de suínos, localizada no planalto médio do Rio Grande do Sul, sendo utilizadas 604 fêmeas de diferentes ordens de parto e com, no máximo, sete dias de intervalo desmame-estro. Após o desmame, as fêmeas foram alojadas em gaiolas e submetidas a dois diagnósticos de estro diários (às 9:00 e 16:00 horas). Um macho sexualmente maduro era conduzido pelo corredor central da instalação (em frente às celas das fêmeas), proporcionando um contato naso-nasal com as matrizes, enquanto um funcionário testava todas as fêmeas para a apresentação do reflexo de tolerância ao homem na presença do macho (RTM). Na primeira apresentação do RTM, as fêmeas eram imediatamente transferidas para a sequiência de gestação, onde eram alojadas em celas, para a realização da IA. O diagnóstico dos retornos ao estro foi efetuado uma vez por dia a partir do $18^{\circ}$ dia após a IA utilizando um macho sexualmente maduro.

Após a manifestação do estro pós-desmame ou estro da cobertura, os animais foram distribuídos em três tratamentos, levando em consideração a ordem de parição, média de leitões nascidos totais nos partos anteriores, duração da lactação e intervalo desmameestro.

No tratamento 1 (T1 ou método auto IA), as fêmeas foram inseminadas com a utilização de pipetas longas lameladas e descartáveis, do tipo Supertip ${ }^{\circledR}$ e o sêmen acondicionado em flexitubos ${ }^{\circledR}$ de espessura fina, que permitia o colabamento do frasco ao esvaziamento, e o aparato de IA do método auto IA. O aparato de IA era constituído por duas partes: a primeira, uma cinta abdominal com regulagem de abertura, uma porção livre com velcro para fixar a cauda e um cordão para sustentar a pipeta de IA na posição vertical, durante a IA; a segunda parte, constituída de uma mala dorsal com pesos laterais (10$12 \mathrm{~kg}$ no total), colocada sobre o dorso da fêmea durante a IA. No tratamento 2 (T2 ou método Intermediário) as fêmeas foram inseminadas com pipetas do tipo Supertip ${ }^{\circledR}$, e o sêmen acondicionado em flexitubos ${ }^{\circledast}$ de espessura fina, sem utilização do aparato de IA, sendo que a DI foi mantida na posição vertical e em nível mais elevado que o trato reprodutivo da fêmea pelo próprio funcionário durante a execução da IA. No tratamento 3 (T3 ou método Tradicional), as matrizes foram inseminadas utilizando pipetas do tipo Melrose, com o sêmen acondicionado em bisnagas e sem qualquer aparato de IA, ficando o tempo de inseminação atrelado à pressão exercida pelo inseminador sobre a DI durante a IA. 
As inseminações foram realizadas em turnos subseqüentes ao diagnóstico de estro com intervalos de 7-17 horas desde que as fêmeas ainda apresentassem o RTM, seguindo o protocolo de IA adotado pela granja, ou seja, entre 3 e 4 IAs no total, embora seja conhecido que com 2 ou, no máximo 3 IAs, os resultados de desempenho reprodutivo sejam semelhantes aos protocolos que utilizam mais IAs por estro.

As doses inseminantes (DIs) acondicionadas em flexitubos ${ }^{\circledR}$ ou bisnagas continham um volume de $100 \mathrm{~mL}$ e 4 bilhões de espermatozóides, diluídos em BTS e armazenadas a $15-18^{\circ} \mathrm{C}$ por, no máximo, 48 horas após a coleta, sendo utilizadas somente as que apresentavam no mínimo $70 \%$ de espermatozóides móveis no momento da IA.

Os refluxos durante a IA e até 120 minutos após, foram coletados de 108 fêmeas pluríparas distribuídas em T1 $(n=41), T 2(n=35)$ e T3 $(n=32)$, sendo os animais escolhidos ao acaso. O número de fêmeas utilizado foi determinado em função do tempo disponível para a coleta do refluxo, durante a realização do experimento. Foram realizadas medidas do volume de refluxo da DI durante as inseminações e aos 120 minutos, cronometrados os tempos de infusão da DI e realizadas estimativas do grau de dificuldade apresentado para a infusão da DI. Para a avaliação do volume do refluxo no momento das IAs foram utilizados copos graduados que foram mantidos sob a rima vulvar durante todo o tempo de IA até o momento da retirada da pipeta. Para as 108 fêmeas que tiveram os refluxos pós-IAs coletados por 120 minutos, utilizaram-se bolsas de colostomia fixadas na região peri-vulvar, logo após a retirada da pipeta de inseminação. O refluxo total coletado aos 120 minutos mais o refluxo colhido no momento da IA, foi mesclado e homogeneizado, pesado para a verificação do volume e retirada uma alíquota para contagem de células espermáticas, em câmara hemocitométrica tipo Neubauer improved ${ }^{\circledR}$.

Para a estimativa do grau de dificuldade para infundir a DI, foi quantificado o número de intervenções (readaptação da pipeta, rearmação do sistema de sustentação da DI ou o aparato de IA) que o funcionário realizava durante a IA, ou seja, quantas vezes foram necessárias intervenções do inseminador para completar a IA. O valor "zero" representou a ausência de intervenção direta do funcionário na IA e os graus de dificuldade 1 e 2 representaram o número de intervenções efetuadas. $\mathrm{O}$ grau de dificuldade 3 representou as inseminações com mais de duas intervenções, ou aquelas que não teriam se realizado sem a intervenção do funcionário durante a IA.

O desempenho reprodutivo foi avaliado pela taxa de retorno ao estro (TRE), taxa de parto ajustada (TPA), que foi calculada a partir da taxa de parto, porém desconsiderando aquelas fêmeas descartadas por problemas não reprodutivos e as que morreram, e pelos leitões nascidos totais (LNT) (leitões nascidos vivos, mortos e mumificados) e vivos (NV). Não foi considerado na análise dos dados de TRE e TPA em cada ordem de parto, uma vez que o baixo número de repetições dificultaria a interpretação dos resultados.

Os dados de TPA, a TRE e o número de até uma intervenção durante a IA foram analisados pelo teste do Qui-Quadrado (Procedimento FREQ, SAS, 1998). O tamanho da leitegada e o tempo de IA foram analisados pela análise da variância (Procedimento GLM, SAS, 1998), considerando os efeitos dos tratamentos e das ordens de parto no modelo estatístico. As comparações entre as médias foram realizadas pelo teste $\mathrm{t}$ de Student. $\mathrm{O}$ volume de sêmen e o número de espermatozóides refluído pós-IA foram analisados pelo teste não paramétrico de Kruskal-Wallis (Procedimento NPAR1WAY, SAS, 1998).

\section{RESULTADOS}

Os tempos médios de IA foram diferentes nos tratamentos $(\mathrm{P}<0,02)$, com o $\mathrm{T} 1$ apresentando tempo duas vezes menor para a completa infusão da DI em relação ao T3, enquanto o T2, demandou $40 \%$ do tempo de infusão do T3, sendo $23 \%$ mais lento que T1 (Tabela 1).

Com relação ao refluxo de sêmen durante a IA, pode ser observado que o valor zero de mediana, o qual corresponde à ausência de refluxo, foi o valor mais comumente verificado (Tabela 1). Entretanto, na interpretação dos valores da variabilidade dos dados, representados pela amplitude, verificou-se que, em algumas inseminações, foram eliminados de 65 (T1) a $80 \%$ (T2 e T3) do volume contido na DI. Das 108 fêmeas que tiveram os refluxos coletados até 120 horas após a IA, foi observado maior refluxo no T1 que no T2 e T3 $(\mathrm{P}<0,02)$ (Tabela 1), entretanto, observa-se uma grande variação desta característica em todos os tratamentos. Os valores de mediana indicam perdas em volumes superiores a $70 \%$ da DI em todos os tratamentos. Embora os valores de volume de refluxo aos 120 minutos tenham sido maiores e diferentes para o T1 em relação ao T2 e T3 $(\mathrm{P}<0,02)$, o mesmo não foi verificado no número de espermatozóides eliminados no refluxo aos 120 
Tabela 1 - Duração da insedminação, volume de refluxo durante a aplicação da inseminação, volume total e número de espermatozóides (SPTZ) e percentual da dose (\% dose) eliminados por refluxo até 120 minutos.

\begin{tabular}{|c|c|c|c|}
\hline & $\mathrm{T} 1$ & $\mathrm{~T} 2$ & $\mathrm{~T} 3$ \\
\hline \multicolumn{4}{|c|}{ Tempo de Inseminação em minutos } \\
\hline Fêmeas (n) & 199 & 207 & 198 \\
\hline $\mathrm{N}^{\mathrm{o}}$ de Inseminações & 730 & 760 & 751 \\
\hline \multicolumn{4}{|l|}{ Tempo de IA (minutos) } \\
\hline Média \pm DP & $1,7^{\mathrm{a}} \pm 1,6$ & $2,2^{\mathrm{b}} \pm 1,8$ & $3,6^{\mathrm{c}} \pm 1,1$ \\
\hline Amplitude (minutos) & $0,2-10,1$ & $0,2-13,7$ & $0,6-9,7$ \\
\hline \multicolumn{4}{|c|}{ Volume de refluxo durante a IA } \\
\hline Fêmeas (n) & 199 & 207 & 198 \\
\hline $\mathrm{N}^{\mathrm{o}}$ inseminações & 730 & 760 & 751 \\
\hline \multicolumn{4}{|l|}{ Volume refluxo $(\mathrm{mL})$} \\
\hline Média \pm DP & $7,7 \pm 13,5$ & $6,7 \pm 13,5$ & $5,8 \pm 10,8$ \\
\hline Mediana (mL) & 0 & 0 & 0 \\
\hline Escores Médios* & $1167,62^{\mathrm{a}}$ & $1096,29^{\mathrm{ab}}$ & $1133,14^{\mathrm{b}}$ \\
\hline Amplitude do refluxo (mL) & $0,0-65,0$ & $0,0-80,0$ & $0,0-80,0$ \\
\hline \multicolumn{4}{|c|}{ Volume total de refluxo até 120 minutos } \\
\hline Fêmeas (n) & 41 & 35 & 32 \\
\hline $\mathrm{N}^{\mathrm{o}}$ de inseminações & 143 & 130 & 117 \\
\hline \multicolumn{4}{|l|}{ Volume refluxo (mL) } \\
\hline Média $\pm \mathrm{DP}$ & $73,5 \pm 21,5$ & $66,0 \pm 23,2$ & $65,0 \pm 26,7$ \\
\hline Mediana (mL) & 76,0 & 70,5 & 71,0 \\
\hline Escores Médios* & $218,32^{\mathrm{a}}$ & $182,79^{b}$ & $182,72^{\mathrm{b}}$ \\
\hline Amplitude (mL) & $0-118$ & $0-110$ & $0-109$ \\
\hline Dose $(\%)$ & 73,5 & 66,6 & 65,1 \\
\hline \multicolumn{4}{|c|}{ Número de espermatozóides eliminados no refluxo até 120 minutos } \\
\hline Fêmeas (n) & 41 & 35 & 32 \\
\hline $\mathrm{N}^{\mathrm{o}}$ de Inseminações & 143 & 130 & 117 \\
\hline \multicolumn{4}{|l|}{$\mathrm{N}^{\mathrm{o}}$ espermatozóides (bilhões) } \\
\hline Média \pm DP & $1,3 \pm 0,7$ & $1,2 \pm 0,6$ & $1,1 \pm 0,6$ \\
\hline Mediana (mL) & 76,0 & 70,5 & 71,0 \\
\hline Escores médios* & $209,62^{\mathrm{a}}$ & $192,15^{\mathrm{a}}$ & $181,95^{\mathrm{a}}$ \\
\hline Amplitude (bilhões de SPTZ) & $0-3,9$ & $0-4,2$ & $0-2,7$ \\
\hline Dose $(\%)$ & 32 & 30 & 28 \\
\hline
\end{tabular}

Letras diferentes na mesma linha, $\mathrm{P}<0,02$.

Letras iguais na mesma linha, $\mathrm{P}>0,05$

* Rankings médios de cada tratamento oriundos da análise não paramétrica de Kruskal-Wallis.

minutos ( $\mathrm{P}>0,05$; Tabela 1$)$ apresentando quantidades semelhantes de espermatozóides eliminados. Em todos os tratamentos, foram verificadas inseminações com ausência de espermatozóides no refluxo.

$\mathrm{Na}$ figura 1, está representado o grau de dificuldade estimado em cada tratamento para a execução da IA obtidos da avaliação de 604 fêmeas inseminadas. O tratamento 1 , que correspondeu ao maior grau de automação, apresentou os maiores valores percentuais de intervenções $(33,6 \%)$, contra $19 \%$ e $7,7 \%$ de intervenções para o T2 e T3, respectivamente. Verifica-se que $85,7,92,9$ e $97,7 \%$ das inseminações em T1, T2 e T3, respectivamente, foram efetuadas com no máximo uma intervenção, 
sendo estes percentuais diferentes entre os três tratamentos $(\mathrm{P}<0,05)$.

Para a análise da TRE, foram utilizadas 586 fêmeas, tendo sido retiradas 18 fêmeas da análise (5, 3 e 10 fêmeas em T1, T2 e T3, respectivamente) por descarte e mortes no período entre a IA e 18 dias póscobertura. Não foram verificadas diferenças $(\mathrm{P}>0,05)$ entre os tratamentos para a variável retorno ao estro. Independentemente do método ou tratamento aplicado, não foram observadas diferenças $(\mathrm{P}>0,50)$ no número de leitões nascidos totais, vivos ou na taxa de parto ajustada entre os tratamentos (Tabela 2).

\section{DISCUSSÃO}

No presente estudo, foi verificada uma diferença significativa entre os tratamentos $(\mathrm{P}<0,02)$ no que se refere ao tempo necessário para a aplicação da DI. A diferença verificada não teve relação com as categorias de OP dentro dos tratamentos. Os flexitubos ${ }^{\circledR}$ empregados no T1 e T2, por serem de espessura fina e macia, permitem o colabamento à medida que o sêmen flui para o trato reprodutivo. Em contrapartida, as bisnagas utilizadas no T3 não permitem o colabamento, havendo a necessidade de pressão sobre as mesmas e, desta maneira, o tempo de IA no T3 ficou atrelado à recomendação técnica da IA tradicional, ou seja, não menos de 4 minutos por IA. A amplitude de variação observada na auto IA no presente experimento, é semelhante àquela observada por MARTIN RILLO et al. (1998), que encontraram variações entre 2,3 vezes menos e 2,7 vezes mais tempo para execução da IA. A redução do tempo de IA no $\mathrm{T} 1$ e $\mathrm{T} 2$, respectivamente de 53 e $38 \%$, comparado ao T3, mostra que a proposta de redução do tempo de IA é efetiva, o que permite um ganho operacional considerável no trabalho de uma granja. A capacidade de fêmeas atendidas por funcionário por hora, utilizando o método da auto IA, pode ser triplicada (HANDS..., 2002).

Tabela 2 - Distribuição dos retornos regulares ao estro (TRE), taxa de parto ajustada (TPA), leitões nascidos totais (LNT) e nascidos vivos (NV) nos tratamentos (T)*.

\begin{tabular}{|c|c|c|c|c|c|}
\hline $\mathrm{T}$ & $\mathrm{IA}(\mathrm{n}$ & TRE\% & ТPA\% & LNT(média $\pm \mathrm{DP})$ & NV (média $\pm D P)$ \\
\hline $\mathrm{T} 1$ & 194 & 10,3 & 90,7 & $10,9 \pm 0,4$ & $10,3 \pm 0,4$ \\
\hline $\mathrm{T} 2$ & 204 & 7,4 & 92,6 & $11,1 \pm 0,4$ & $10,4 \pm 0,3$ \\
\hline $\mathrm{T} 3$ & 188 & 8,5 & 90,9 & $11,1 \pm 0,4$ & $10,4 \pm 0,4$ \\
\hline
\end{tabular}

* Não houve diferença entre os tratamentos $(\mathrm{P}>0,05)$.
MARTIN RILLO et al. (1998), analisando as possíveis causas da não absorção da DI no sistema auto IA, concluíram que a obstrução da pipeta na cérvix ou a ausência de contração uterina, foram as principais causas do aumento da duração da inseminação, e que, em ambos os casos, a intervenção do funcionário foi imprescindível, seja manipulando a pipeta ou estimulando mais intensamente a fêmea. No presente trabalho, o volume de refluxo observado durante a IA foi diferente $(\mathrm{P}<0,02)$ entre o T1 e T3, sendo que o T2 não diferiu do T1 e T3. Eventualmente, os refluxos, durante a IA no T1 e T2, puderam ser verificados seguidos da incorreta fixação e/ou, provavelmente, cérvix demasiadamente relaxada, a qual não oferecia uma condição ótima de fixação da pipeta e, dessa forma, levando a episódios de refluxos. Entretanto, o grande diferencial entre T1 e T2 para o T3 foi a ausência de controle do tempo de infusão da DI, pelo fato de o tempo de aplicação da DI nesses tratamentos, ter ficado atrelado à capacidade e contratilidade uterina da fêmea. Em alguns casos, como nos de incorreta fixação da pipeta de IA ou cérvix relaxada, ocorreram inseminações realizadas em curtos espaços de tempo, apresentando, consequentemente, refluxos mais abundantes que os normalmente verificados na média dos tratamentos. LEVIS (2002) sugere que o maior refluxo acontece com o uso deste método, quando a supervisão da IA não é bem feita.

Os volumes de refluxo aos 120 minutos apresentados em T1 e T2, podem ser explicados, em parte, pelas peculiaridades dos tratamentos, como o aparato de estimulação presente no T1 e uso de flexitubos ${ }^{\circledR}$ no $\mathrm{T} 1$ e $\mathrm{T} 2$, e pela própria metodologia aplicada, uma vez que permitiu uma IA em menor espaço de tempo (1,7 e 2,2 minutos em média, para $\mathrm{T} 1$ e $\mathrm{T} 2$ respectivamente) em relação ao T3 (3,6 minutos) e pelo sistema de acondicionamento/embalagem do sêmen em flexitubos ${ }^{\circledR}$ que permitiu a descida da DI oferecendo pouca resistência.

No estudo de STEVERINK et al. (1998), quando somados os volumes de refluxos nos 3 momentos da análise (5, 30 e 150 minutos), foi verificado que o somatório do volume total de refluxo aos 150 minutos foi de $70 \pm 3,4 \%$ da DI, apresentando uma amplitude de 17 a 120\%. Esses valores são muito próximos às médias de refluxo aos 120 minutos, nos três tratamentos, observadas no presente experimento, de $68,7 \%$ da DI, com uma amplitude de 0 a $118 \%$ (mínima e máxima considerando os três tratamentos) do volume da DI. Embora seja um trabalho mais antigo BAKER et al. (1968) observaram na média, perdas 
muito menores ou seja da ordem de 22 a $39 \%$ do volume da DI.

VIRING \& EINARSSON (1981) sugerem que, aproximadamente 1/3 dos espermatozóides do ejaculado, são eliminados por refluxo em 2 horas após a cobertura. No atual estudo, foi verificado que, em média, 32\% (T1), 30\%(T2) e 28\% (T3) dos espermatozóides da DI foram eliminados no refluxo até 120 minutos após a IA ( $\mathrm{P}>0,05)$. No estudo de STEVERINK et al. (1998), quando somados os valores obtidos a partir da avaliação dos refluxos nos 3 momentos da análise (5, 30 e 150 minutos), foi verificado que o somatório do número total de espermatozóides do refluxo aos 150 minutos foi $25 \pm 1,3 \%$ da DI, apresentando uma amplitude de 3 a $48 \%$. Esses valores estão muito próximos aos encontrados no presente experimento, em que foram verificados uma média do total de espermatozóides do refluxo aos 120 minutos, de $30 \%$ da DI, com uma amplitude de 0 a $105 \%$.

Na medida dos graus de dificuldade para a execução das IAs nos tratamentos, foi observado que, no T1, 66,4\% dos animais tiveram as IAs conduzidas sem qualquer tipo de intervenção. Este resultado, quando analisado isoladamente, poderia ser considerado insuficiente, pois a expectativa para um procedimento de maior automação seria a de que houvesse menor grau de intervenção.
Entretanto, como o grau de dificuldade 1 correspondeu, na prática, àquele acompanhamento inicial com uma discreta correção ou ajuste do aparato de IA, pipeta ou DI, não sendo necessárias outras correções, $85,6 \%$ das fêmeas do T1, não teriam apresentado problemas para a execução da IA (Figura 1). Mesmo necessitando de um certo grau de intervenção, o sistema automatizado teria a vantagem de permitir a inseminação simultânea de um determinado número de fêmeas, bastando que o operador estivesse atento para a eventual necessidade de pequenos ajustes, sem que houvesse uma dedicação exclusiva à inseminação de cada fêmea. Os resultados de aceitação do método foram similares aos descritos por MARTIN RILLO et al. (1998) com o método auto IA, uma vez que nenhuma fêmea em estro recusou o aparato utilizado na IA do T1. No presente estudo, 85,6\% das fêmeas no T1 apresentaram graus de dificuldade zero e 1, com menor grau de intervenção do que a citada por MARTIN RILLO et al. (1998) para o método auto IA. Este resultado pode ser justificado, em parte, porque no presente experimento foram avaliados um maior número de animais e várias categorias de ordem de parto. Ainda no T1, deve ser salientado que a dificuldade de grau $1(19,2 \%)$ normalmente esteve relacionada com a inadequada colocação/ fixação do aparato de IA, uma vez que, com uma

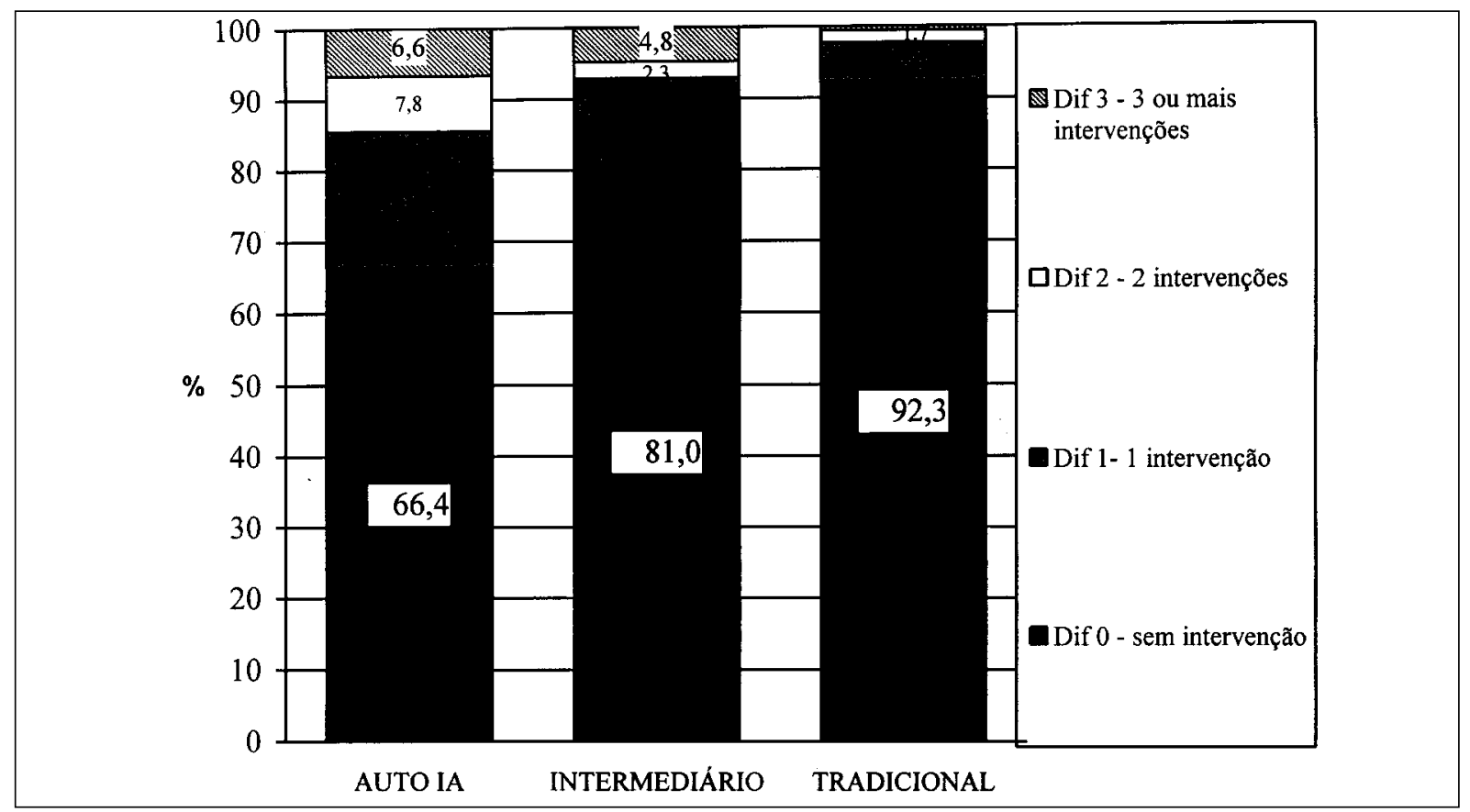

Figura 1 - Representação percentual da estimativa do grau de dificuldade para a execução da inseminação artificial avaliado em cada tratamento. 
única intervenção, houve a correção do problema.

Não ocorreram diferenças entre os tratamentos com relação à taxa de retornos ao estro, taxa de parto ajustada, leitões nascidos totais e vivos (Tabela 2). MARTIN RILLO et al. (1998) observaram desempenho reprodutivo semelhante quando utilizado o método da auto IA.

Pode ser considerado que a tecnologia utilizada de auto IA não apresenta diferenças no desempenho reprodutivo, permitindo uma otimização dos procedimentos da IA e uma melhor utilização da mão de obra altamente treinada.

\section{REFERÊNCIAS BIBLIOGRÁFICAS}

BAKER, R.D.; DZIUK, P.; NORTON, H.W. Effect of volume of semen, number of sperm and drugs on transport of sperm in artificially inseminated gilts. Journal of Animal Science, v.27, p.88-93, 1968

BORTOLOZZO, F.P; WENTZ, I. Incremento da eficiência reprodutiva em programa de inseminação artificial (IA) no suíno. In: CONGRESSO BRASILEIRO DE REPRODUÇÃO ANIMAL,
1995, Belo Horizonte, MG. Anais... Belo Horizonte : Colégio Brasileiro de Reprodução Animal, 1995. p.131-141.

HANDS-free or hands-on. Pig International, v.30, n.3, p.21-22, 2000 .

LEVIS, D.G. Use of hands-free insemination devices. Ohio: Ohio Pork Industry Center, The Ohio State University Extension, 2002. p.55-61. Disponível na Internet: http:// www.porkinfo.asu.edu/levis.htm. Online.

MARTÍN RILLO, S. et al. Hands-free artificial insemination in swine. In: INTERNATIONAL PIG VETERINARY CONGRESS, 15., 1998, Birminghan, England. Proceedings... Birmingham, England : Nothingham University, 1998. V.2, p.34.

SAS INSTITUTE. SAS user's guide: statistical analysis system, Release 6.12. Cary, NC, 1998.

STEVERINK, D.W.B. et al. Sêmen backflow after insemination and its effect on fertilisation results in sows. Animal Reproduction Science, v.54, n.2, p.109-119, 1998.

VIRING, S.; EINARSSON, S. Sperm distribuition within the genital tract of naturally inseminated gilts. Nordisch Veterinarian Medicine, v.33, p.145-149, 1981. 